\title{
Validation of a Physical and Numerical Model to Solve Problems of Seepage Flow
}

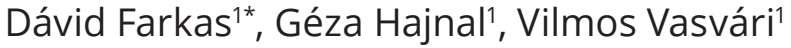 \\ 1 Department of Hydraulic and Water Resources Engineering, Faculty of Civil Engineering, \\ Budapest University of Technology and Economics, \\ Múegyetem rkp. 3, H-1111 Budapest, Hungary \\ * Corresponding author, e-mail: farkas.david@epito.bme.hu
}

Received: 28 May 2018, Accepted: 11 January 2019, Published online: 25 February 2019

\begin{abstract}
The coefficient of permeability ( $k$ value) is an important parameter in civil engineering practice, in hydrology and hydrogeology. It can be determined by field test or by means of laboratory testing. The goal of this paper is to assess this parameter by creating a laboratory model and by validating its results using finite element computer code. The model tests provide that can be applied for estimating permeability of different soils. In a physical model medium-grained sand was tested in the laboratory, for understanding the effects of different flow rates on the validation of the measurement result, the numerical simulation of the physical model was constructed using FEFLOW. Two model variants were developed and both variants were calibrated and validated. Subsequently, the results were converted to real variables based on the model laws. The physical model provides the flow rate of the well in mediumgrained sand with sufficient accuracy if the real size of the drawdown is between 0,5 and 1,7 $\mathrm{m}$.
\end{abstract}

Keywords

pumping test, hydraulic conductivity, model laws, physical model, numerical model

\section{Introduction}

In Hungary, there are no standards and technical guidelines for pumping tests used in the construction industry. The European standard EN ISO 22282 [1] is available but has not been implemented in practice. Due to a high number of field investigations, it has been found that the permeability of a site can be determined more expertly and cheaper if the adequate geological and geotechnical knowledge is available. The essence of the geotechnical practice is that the soil or rock samples are subjected to laboratory tests. Part of these investigations are suitable for determining the permeability coefficient (k value), e.g. in granular soils by examining the grain distribution and by the Darcy test, however, these are disturbed samples. In the field, the best in situ test for determining the $\mathrm{k}$ value is the pumping test. In order to fully understand this experiment, our sand model was built with the aim of not only determining the permeability of a particular soil layer but recognizing the applicability and limitations of this method. Based on this, guidelines for practitioners can be provided even in the case of a few initial data. Simultaneously with the model tests, these were supported by numerical modeling and validated.
Physical models were used in many cases until the 1980s [2] to measure the characteristics of the investigated seepage flow or at least to determine some parameters of the flow describing relationships.

From the 1980s, sand models [3] were increasingly replaced by numerical models, but the former are still used today. With the help of sand models, the following questions and thus types of groundwater flow are usually investigated:

- Inflow to a well [4-6]

- Drawdown of groundwater for foundation pit drainage [7]

- Seepage through and beneath the dam embankments of barrages and protective dikes or sheet piling [8].

- Investigation of density flows [9-10].

Physical and numerical models were also used to observed seepage flow in fractured rocks, not just in porous media [11-13].

First, the model laws for physical sand models based on the work of [14] and [15] are summarized and briefly described. 
It will then be presented by which accuracy the results of the physical model and the numerical modeling are assignable to each other. It is also discussed in which area the transfer of model results to real sizes can be used in practice.

\section{Model laws for hydraulic models}

A hydraulic model is a scale representation of the flow space in which the hydrodynamic process is investigated.

Three prerequisites must be met for complete hydromechanical similarity: geometric similarity, kinematic similarity, and dynamic similarity. Accordingly, three conversion factors can be defined between the prototype and the model. These are the length scale $\lambda$.

$\frac{l}{l^{\prime}}=\lambda=$ const

$l$ - length in the prototype, $l^{\prime}$ - length in the model, the time scale $\tau$

$\frac{t}{t^{\prime}}=\tau=$ const

$t$ - time in the prototype, $t^{\prime}$ - time in the model, as well as the force scale $\pi$

$\frac{F}{F^{\prime}}=\pi=$ const

$F$ - force in the prototype, $F^{\prime}$ - force in the model.

The most important forces acting within the flow field are gravity $(\mathrm{G})$, internal friction $(\mathrm{R})$ and inertia $(\mathrm{T})$. Even in the case of other hydrodynamic processes, these forces are considered dominant effects. Three forces provide three equations expressing the relationship of the forces corresponding within the two connected systems [14].

The most common case is when the two liquids (mostly water) are the same. If the differences in density and viscosity caused by the possible temperature difference are neglected, the equations provide the following conditions for the relationship between the three model scales $\lambda, \tau$ and $\pi$, assuming that only the mentioned three forces are dominant in the system:

$$
\begin{aligned}
& \varphi_{G}(\lambda, \tau, \pi)=\lambda^{3}-\pi=0 \\
& \varphi_{R}(\lambda, \tau, \pi)=\frac{\lambda^{2}}{\tau}-\pi=0 \\
& \varphi_{T}(\lambda, \tau, \pi)=\frac{\lambda^{4}}{\tau}-\pi=0
\end{aligned}
$$

The solution of this system of equations provides the simplified result:

$\lambda=\tau=\pi=1$

which states that in the case where the same fluid is used both in the prototype and in the model and three or more dominating forces occur whose effect can not be neglected, the full hydromechanical similarity can only be achieved if the geometric extents of the two systems and of course the corresponding time periods and the related forces are also the same.

The use of small scale models using the same liquid is limited to the case where the influence of only two forces is dominant in the system and therefore the impact of others can be neglected or their effect can be considered in a special way.

The similarity secured by the consideration of only two main forces is considered to be partial mechanical similarity, and the well-known model laws always provide the relationship between $\lambda, \tau$ and $\pi$ [15].

\section{The Mosonyi-Kovács number}

If only two main forces are dominant, two conditions from Eqs. (4) to (6) must be considered simultaneously. One of the basic scales can be arbitrarily selected (mainly that of length, $\lambda$ ) and the other two can be calculated as a function of it. Knowing this relationship, the scale of the other characteristic quantities can be expressed as a function of the value $\lambda$.

On the basis of the dynamic analysis of the seepage process, it is well known that the dominant role of gravity and friction in laminar seepage is characteristic. The theoretical derivation of the Darcy Law is also based on this hypothesis. The dimensionless number, which is the quotient of friction and gravity, is the Mosonyi-Kovács number MK [15-16]:

$\frac{v v}{l^{2} g}=\frac{v^{\prime} v}{l^{\prime 2} g}=M K=$ const .

$v$ and $v^{\prime}$-seepage velocity in the prototype and in the model, $v$ - kinematic viscosity; $l$ and $l$ ' characteristic length (pore diameter) in the prototype and in the model, $g$ - acceleration of gravity.

When examining this type of motion, the relationship between the basic scales can be expressed by the following formula:

$\tau=\frac{1}{\lambda}$ and $\pi=\lambda^{3}$. 
In view of the analysis of the model laws, it can be stated that the practically realisable mechanical similarity in two geometrically similar seepage spaces is ensured when the Mosonyi-Kovács number calculated from the corresponding data of the two systems is constant. In this case, all sizes of the two systems, not only the lengths of the dimensions but also the grain sizes are proportional to each other, that is, $\lambda$ is constant for each size.

\section{Geometrically distorted sand models}

As explained in the previous chapter, only a partial mechanical similarity between the prototype and the hydraulic model can be achieved. The process on a small scale may differ significantly from the original system if the effect of the disregarded forces cannot be neglected. Therefore, the features calculated from the data observed in the model may differ from the actual parameters. It is obvious that better results can be achieved if the size of the model is closer to that of the prototype because in this case the effects of the neglected forces in both systems become more and more similar. This phenomenon is called scale effect. If the model size moves in the other direction as the ratio of the corresponding geometric parameters increases, then the differences caused by the change in the neglected forces become larger and larger. There is a certain limit and if the model is scaled down below, the data measured in the small-scale model can not be used to determine the actual parameters because of the uncertainties arising from the different effects of the neglected forces. It is therefore necessary to add another limit to the previous one when investigating the seepage flow using a small scale sand model.

To ensure the geometric similarity, each size of the prototype must be reduced in the same proportion as for the length scale determined. This proportionality should also be ensured between the grain sizes of the two systems. It is obvious that the reduction of the grain size of the soil matrix used in the model below a certain limit is physically impossible. The high proportion of colloid particles can cause the change in the physical character of the seepage process [15].

If the existing construction requires a smaller model than that given by one of the limits, the general process is to distort one or more geometric parameters. In sand models, the geometry of the boundary conditions of the flow space is always proportionally reduced, but the grain size is distorted by the use of coarser particles than these, which would be necessary based on geometrical similarity.
This is the usual practice of filling up the flow space of the sand model with the same material as that of the prototype. To use the original porous medium in the model is a special case of distorted model if the scale of the grain size is equal to the unit $\left(\lambda_{\mathrm{D}}=1\right)$.

\section{Physical model}

\subsection{Determination of the model geometry}

The physical model was created in the laboratory taking into account the model laws discussed in the previous chapters. At first it was necessary to define the geometric parameters. Pumping tests cause a cylindrically symmetric flow in a homogeneous medium, so the model should be created with the outline of a circle.

Determining the actual expansion of the cone of depression in nature is difficult for both technical and theoretical reasons. The cone of depression can vary in several orders of magnitude, depending on whether the pumping test is carried out in fine-grained or coarse-grained soils. For this reason, the geometric dimensions were determined not by the cone of depression, but by the diameter of the well and the drawdown in the well.

The geometrical model scale and thus the model dimensions were finally determined with regard to the manageability of the model in the laboratory. The starting point was a conventional filter tube diameter of $160 \mathrm{~mm}$, a drawdown of up to $2 \mathrm{~m}$ and a thickness of the aquifer of $12 \mathrm{~m}$. These geometric parameters give the following dimensions in the model for a chosen scale of $\lambda=16$ : filter pipe diameter $10 \mathrm{~mm}$, expected drawdown to $12.5 \mathrm{~cm}$ and a thickness of $75 \mathrm{~cm}$. When choosing the length scale, the realization of the well in the model was crucial. A minimum diameter of the delivery well of $10 \mathrm{~mm}$ was required in order to be able to pump out the corresponding quantities from the model. The well was initially fully penetrated.

Table 1 Conversion factors of the most important physical quantities according to the MK number (according to [15])

\begin{tabular}{|c|c|c|c|c|}
\hline \multirow{3}{*}{$\begin{array}{l}\text { Physical } \\
\text { quantity } \\
\text { Length }\end{array}$} & \multicolumn{4}{|c|}{ Conversion factor according to the MK number } \\
\hline & \multicolumn{2}{|c|}{ undistorted } & \multicolumn{2}{|c|}{ distorted } \\
\hline & 1 & 16 & 1 & 16 \\
\hline Area & 12 & 256 & 12 & 256 \\
\hline Volume & 13 & 4096 & 13 & 4096 \\
\hline Time & $1-1$ & $1 / 16$ & 1 & 16 \\
\hline Velocity & 12 & 256 & 10 & 1 \\
\hline Acceleration & 13 & 4096 & $1-1$ & $1 / 16$ \\
\hline Discharge & 14 & 65536 & 12 & 256 \\
\hline
\end{tabular}




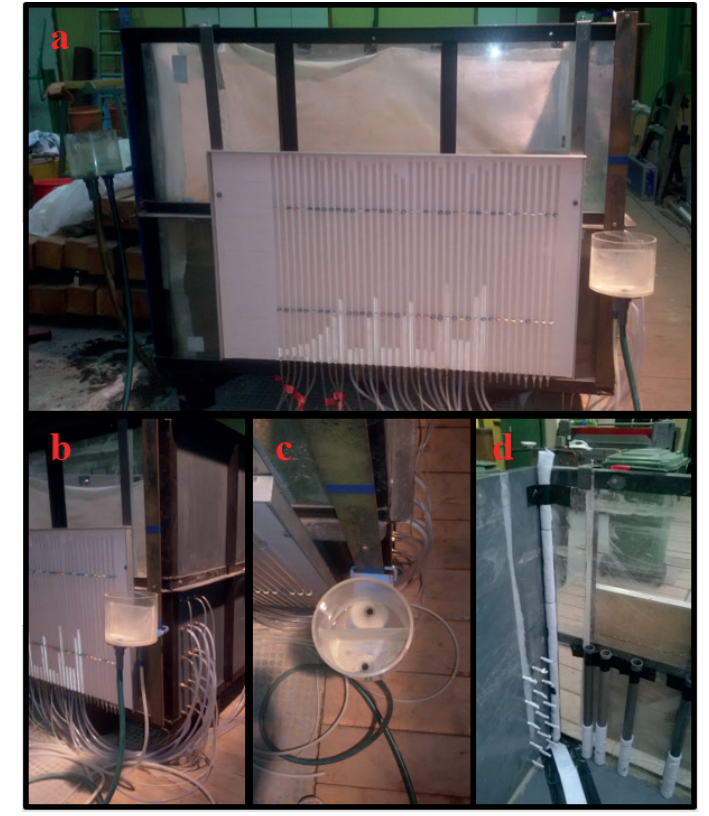

Fig. 1 Side view of the physical model with the weir overflows for supply (left) and extraction (right) as well as the piezometer harp (a), side view of the weir overflow of the extraction (b) top view of the weir overflow of the extraction (c), design of the piezometers, the pumping well and the water level gauges in the unfilled model (d)

After determining the geometrical model scale, the conversion factors of the other physical quantities were determined according to the model law of Mosonyi-Kovács (Table 1), whereby in the realized model the conversion factors of a distorted model were used.

At a distance of $7 \mathrm{~cm}$ from the well (piezometer P1), the condition $\operatorname{Re}<1$ is fulfilled, so that the seepage flow is laminar.

\subsection{Setup of the physical model}

The physical model (Fig. 1) was therefore built with the following dimensions:

- Horizontal section: circular sector with $90^{\circ}$

- Height: $1.00 \mathrm{~m}$

- Radius up to the recharge area: $1.28 \mathrm{~m}$

- Total radius: $1.335 \mathrm{~m}$

- Volume: $1.4 \mathrm{~m}^{3}$

- Weight: $100 \mathrm{~kg}$

- Diameter of the pumping well: 10 or $17 \mathrm{~mm}$.

In our model, it is allowed to use the shape of a quarter-circle sector because previous studies [17-18] have shown that the shape of the model does not distort the results of the investigations. Previous studies have also shown that the physical model is suitable for determining the permeability coefficient, since the values obtained correspond to those in the literature. In these measurements, the detection of the drawdown curves was registered by means of 10 piezometers set up on the base plate. The intake of the water into the model space was carried out by a weir overflow and the outflow was regulated by a pump. The recharge area was separated from the sandfilled model domain by a geotextile screened grid.

During the investigations several problems came to light. With the pumping wells used at the beginning of the model tests (diameter $10 \mathrm{~mm}$ ), the required drawdown could not be produced, so it was exchanged for one with a diameter of $17 \mathrm{~mm}$. The well was also surrounded with geotextile. The constant and long-term pumping with the selected pump could not initially be ensured.

Due to the difficulties [19], changes were made to the model. Weir overflows were used not only in the inlet area but also at the extraction. The drawdown was caused by the difference in height of the water levels of the weir overflows. Thus, the uniform extraction was ensured. To read off the pressure in the well an outlet for a piezometer was created. The number of piezometers has been increased from ten to thirty. The piezometers were $5 \mathrm{~cm}$ long, geotextile-coated perforated metal pipes.

Of the newly installed piezometers, 15 were located on the side wall of the model near the pumping well, in three columns at a distance of $5 \mathrm{~cm}$ from each other. In this way, detailed information about the pressure conditions in the immediate vicinity of the well can be recorded. Another five piezometers were also connected one below the other between the side wall and the symmetry axis of the model at a distance of $73 \mathrm{~cm}$ from the well. Here, five piezometers were placed on the plexi-wall of the model at a distance of $15,20,30,45$ and $70 \mathrm{~cm}$ from the well. The observation wells were perforated and geotextile-clad tubes to avoid the wash-in of the fine fraction. These observation wells acted as linear observation wells. The exact position of the piezometers and the observation wells are shown in Fig. 2.

\section{Execution of the model experiments}

The soil examined in the model space corresponds to medium sand according to its grain distribution line. The largest grain diameter of the sand is $\mathrm{d}_{\max }=1 \mathrm{~mm}$, its modal value amounts to $\mathrm{d}_{\mathrm{Mo}}=0.25 \mathrm{~mm}$.

The soil used in the model is not an in-situ sediment. The reason for this is that in order to investigate the process of the pumping test and the applicability of the physical model, it was first necessary to have a completely homogeneous, well compacted soil. Thus, the measurement results influencing effect of heterogeneity existing in 


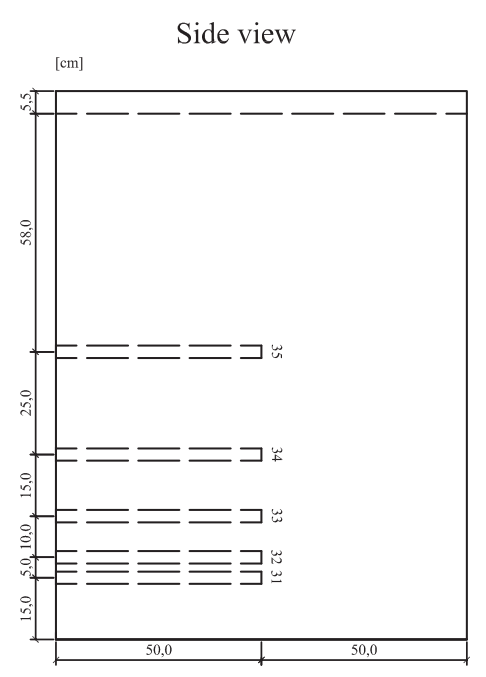

$3 \mathrm{D}$ view

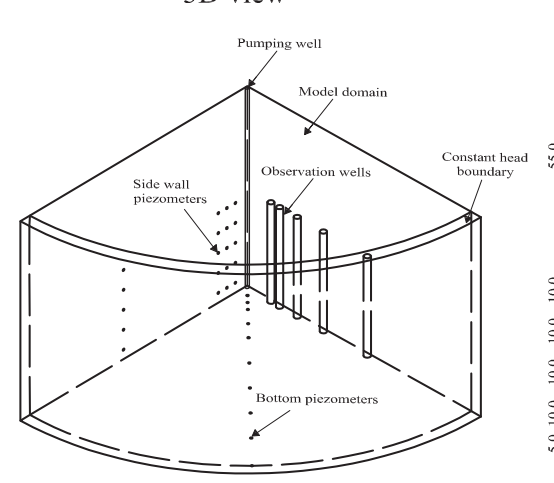

Fig. 2 Setup of the physical model, location of the piezometers and the water level gauges
Top view

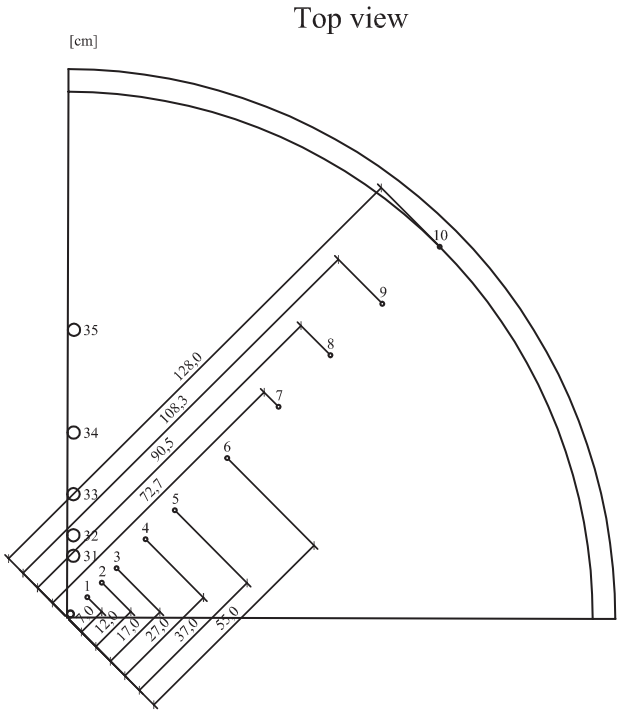

Front view

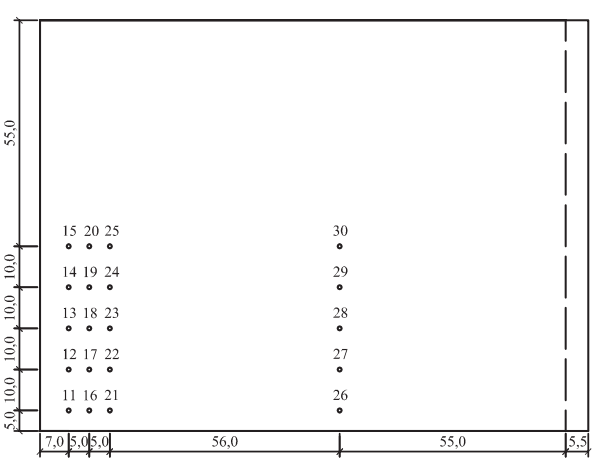

in-situ soil samples can be minimized. Our method had to be verified for a simple soil in order to be able to examine composite soil profiles.

The compaction of the soil was done manually with the aim of uniformity, although a perfectly homogeneous sample cannot be made. The saturation of the soil was carried out several times, with the minimization or complete removal of the air bubbles was sought.

Klotz [20] showed that the existence of air bubbles can cause a deviation of about $30 \%$ in permeability. The permeability coefficients determined by Klotz stabilized after about 1.5 months. In our experiments, about 4 months passed between the last saturation and the measurements. Thus, it can be assumed that air bubbles removable at atmospheric pressure have already been expelled from the system.

In the model, a flow of water was created with the help of two weir overflows positioned at different heights. The water flows into the recharge area of the model via the weir at the inlet. The recharge area forms the zone between the geotextile-clad metal grid and the model bounding arched plate and at the same time acts as the boundary condition of the model. This ensured that the water penetrates the soil uniformly and with constant pressure along the edge of the model. Before the investigations, the soil was saturated, so that the air bubbles completely removed from the model. After the setting of the weir overflow levels, a steady state flow occurred in about 30 minutes. Subsequently, the reading of the pressure levels at the piezometer harp and the water level in the observation wells were started. After reading the pressure levels, the flow of the pumping well was measured three times by means of a stopwatch and measuring cylinder and an average value was calculated. After completion of the measurements, the model for the next experiment was prepared by changing the water level of the weir overflow at the inlet and/ or outlet side and waiting again for the steady state. The model has no heat insulation. The temperature difference between the water fed from the water pipe and the water leaving the model was not registered or neglected. 


\section{Evaluation of the measurement results according to Dupuit}

The permeability coefficients were calculated from the measured data using the Dupuit formula [15].

$$
k=\frac{Q}{\pi} \frac{\ln \frac{r_{1}}{r_{2}}}{h_{1}^{2}-h_{2}^{2}}
$$

$r_{1}$ - distance of the observation well 1 from the pumping well (closer to the well) [m],

$r_{2}$ - distance of the observation well 2 from the pumping well [m],

$h_{1}$ - water level at the observation well $1[\mathrm{~m}]$,

$h_{2}$ - water level at the observation well 2 [m].

$Q$ - flow rate/discharge $\left[\mathrm{m}^{3} / \mathrm{s}\right]$.

The method of Dupuit assumes that the streamlines can be considered horizontal and the vertical component of the velocity vectors can be neglected [15]. The permeability coefficient for the overall model was determined as the mean of the values calculated for the pairs of observation wells. Finally, 15 measurements were averaged and a plausible permeability coefficient of $2.93 \times 10^{-4} \mathrm{~m} / \mathrm{s}$ was determined for the medium sand.

\section{FE model setup in Feflow and calibration}

The model area of the shape of a quarter-circle could be completely covered with the triangular elements used in Feflow [21-22]. The refining of the triangular mesh was reduced radially from the well to allow the drawdown process to be simulated accurately enough in the vicinity of the well. The edge length of the triangular elements in the vicinity of the well was $1 \mathrm{~mm}$, while in the region of the recharge (on the circular arc) it was defined as $2 \mathrm{~cm}$.

The distance between the vertically mounted piezometers is $10 \mathrm{~cm}$ in the physical model. The vertical element density of the numerical model was chosen at half of this interval. In the numerical model 10 model layers with 5 $\mathrm{cm}$ thickness each were defined. Furthermore, 30 piezometers and 5 observation wells were set as observation points in the model. The water level of the recharge area was defined as fix potential on the cylinder jacket. The well was not defined as an extraction, but with its lowered, steady state water level as a fix potential as well.

The initial value of the permeability coefficient for calibration was the mean value of $2.93 \times 10^{-4} \mathrm{~m} / \mathrm{s}$ obtained by the Dupuit method. With the initial permeability value no satisfactory results could be achieved. The subsequent calibration of the permeability was carried out taking into account

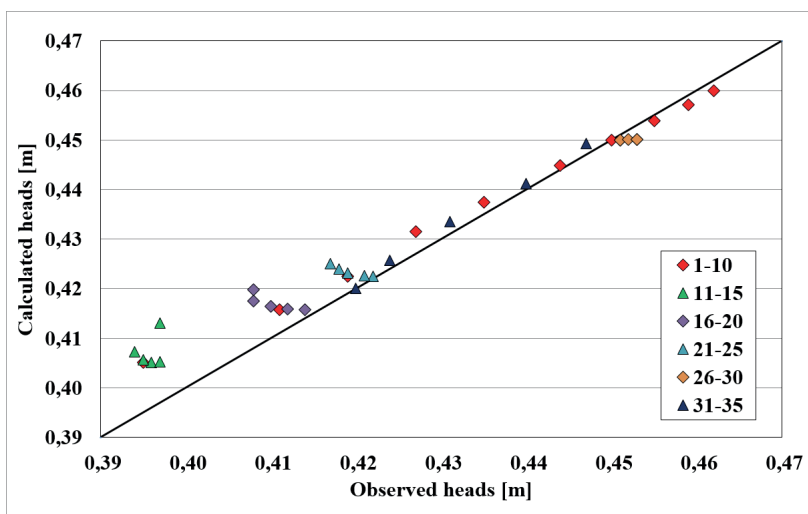

Fig. 3 Calibration results of the homogeneous model

both the measured and calculated hydraulic heads, as well as the flow rates. With a permeability of $2.93 \times 10^{-4} \mathrm{~m} / \mathrm{s}$ in the overall model, a mean absolute error of $4.55 \mathrm{~mm}$ is calculated (Fig. 3).

According to the theory of Dupuit his formula can be used if the produced drawdown is not significant. In our case, there was a considerable drawdown in comparison with the model dimensions, so that the Dupuit theory in the immediate vicinity of the well is no longer valid. In Fig. 3 it can be seen that with increasing distance from the well, where the drawdown becomes negligible compared to the model dimensions, the points have better fitting. So in the homogeneously assumed model, the angular difference between the straight line defined by the measured hydraulic heads and the calibration line remained the same and the larger deviation occurred towards the well.

\section{Analysis of the permeability coefficient distribution 9.1 Model variant $A$}

In the previous chapter it was shown that the soil sample characterizes a certain inhomogeneity both vertically and horizontally, the extent of which increases in the vicinity of the well.

Since the main aim of the experiments was to investigate the pumping test as a process in the physical model, the aim was to calibrate the numerical model with the smallest possible absolute deviation $(\approx 1 \mathrm{~mm})$. For this, the permeability coefficient had to be modified.

To simulate inhomogeneity, the model was divided into several zones to simulate the water level as accurately as possible. When selecting the zone boundaries, the main aspect was to assign the individual piezometers independent subareas. In addition, the area around the well which is most affected by the erosion was treated independently of the piezometers. Because of these aspects, a zone system has been set up. Its detailed parameters are the following: 
Table 2 Distribution of the permeability coefficients used in the model variants

\begin{tabular}{|c|c|c|c|c|c|c|c|c|c|}
\hline \multicolumn{10}{|c|}{ Permeability of zones in model variant A } \\
\hline Skin & $9.26 \mathrm{E}-04$ & II-L1 & 8.77E-05 & III-L1 & $6.24 \mathrm{E}-05$ & IV-L1 & $6.29 \mathrm{E}-05$ & V-L1 & $6.29 \mathrm{E}-05$ \\
\hline \multirow[t]{9}{*}{$\mathbf{I}$} & $2.92 \mathrm{E}-03$ & II-L2 & $1.95 \mathrm{E}-03$ & III-L2 & 1.17E-03 & IV-L2 & $1.89 \mathrm{E}-04$ & V -L2 & $2.05 \mathrm{E}-04$ \\
\hline & & II-L3 & $2.73 \mathrm{E}-04$ & III-L3 & $6.29 \mathrm{E}-05$ & IV-L3 & $1.89 \mathrm{E}-04$ & V -L3 & $1.85 \mathrm{E}-04$ \\
\hline & & II-L4 & $1.85 \mathrm{E}-04$ & III-L4 & $1.56 \mathrm{E}-04$ & IV-L4 & 4.29E-04 & V -L4 & $1.89 \mathrm{E}-04$ \\
\hline & & II-L5 & $9.75 \mathrm{E}-06$ & III-L5 & $5.66 \mathrm{E}-05$ & IV-L5 & $5.66 \mathrm{E}-05$ & V -L5 & $5.66 \mathrm{E}-05$ \\
\hline & & II-L6 & $1.17 \mathrm{E}-04$ & III-L6 & $9.75 \mathrm{E}-05$ & IV-L6 & $1.95 \mathrm{E}-04$ & V -L6 & $5.66 \mathrm{E}-05$ \\
\hline & & II-L7 & $1.95 \mathrm{E}-04$ & III-L7 & $2.92 \mathrm{E}-04$ & IV-L7 & $6.29 \mathrm{E}-05$ & V -L7 & $3.15 \mathrm{E}-04$ \\
\hline & & II-L8 & $3.80 \mathrm{E}-04$ & III-L8 & $1.95 \mathrm{E}-03$ & IV-L8 & $1.89 \mathrm{E}-04$ & V -L8 & $3.15 \mathrm{E}-04$ \\
\hline & & II-L9 & $6.82 \mathrm{E}-05$ & III-L9 & $1.95 \mathrm{E}-03$ & IV-L9 & 4.87E-04 & V -L9 & $3.15 \mathrm{E}-04$ \\
\hline & & II-L10 & $6.24 \mathrm{E}-05$ & III-L10 & 7.31E-05 & IV-L10 & $3.15 \mathrm{E}-04$ & V -L10 & $5.66 \mathrm{E}-04$ \\
\hline \multicolumn{10}{|c|}{ Permeability of zones in model variant B } \\
\hline & & Skin & $\mathbf{I}$ & II & III & IV & $\mathbf{V}$ & & \\
\hline & & $6.07 \mathrm{E}-04$ & $4.05 \mathrm{E}-04$ & $2.45 \mathrm{E}-04$ & $2.45 \mathrm{E}-04$ & $2.45 \mathrm{E}-04$ & $2.45 \mathrm{E}-04$ & & \\
\hline
\end{tabular}

- The implementation of the well was performed in the numerical model with the geometry given above (fully penetrated, $\mathrm{d}=1.7 \mathrm{~cm}$ ). The elements in the well were handled extra and their entirety was defined as the well zone.

- A skin zone with a radial extension of $4 \mathrm{~mm}$ was established around the well. This corresponds approximately to the strength of the geotextile surrounding the well. The skin zone thus surrounds the well vertically in the entire model thickness.

- Zone I comprises a hollow cylinder sector between skin zone and the radius of $5 \mathrm{~cm}$. It goes through the entire model thickness and does not contain any piezometers.

- Zone II comprises a hollow cylinder sector with radii of 5 and $10 \mathrm{~cm}$. The piezometers 1 and 11 to 15 are located in this zone.

- Zone III comprises a hollow cylinder sector with radii of 10 and $15 \mathrm{~cm}$. The piezometers 2 and 16 to 20 are located in this zone.

- Zone IV comprises a hollow cylinder sector with radii of 15 and $20 \mathrm{~cm}$. The piezometers 3 and 21 to 25 are located in this zone.

- Zone $\mathrm{V}$ comprises a hollow cylinder sector with radii of 20 and $128 \mathrm{~cm}$.

Zones II to $\mathrm{V}$ were divided vertically into 10 equal areas (Fig. 2).

The well zone and the skin zone as well as zone I were not vertically structured. It was assumed that the washout of the fine particles in the immediate vicinity of the well took place in its entire vertical length. Since each of the zones II to IV contains superimposed piezometer, which are affected with different errors (Fig. 3), these zones were also divided vertically $(10 \times 5 \mathrm{~cm})$.

From the above, it is apparent that the model was divided into a total of 43 zones. The 43 zones may appear as a high number, but it should be noted that 33 out of 43 zones make up only $2.5 \%$ of the model volume (within a radius of $20 \mathrm{~cm}$ ). The high resolution around the well was considered necessary to accurately approximate the water level, flow pattern and permeability coefficient as much as possible.

The permeability coefficients of the individual zones are shown in Tab. 2. It has been assumed that the washout of the fine grains takes place most strongly in the zone I closest to the well. Therefore, it was noted that the permeability coefficient of the zone I is always greater than that of the more remote zones. During calibration, the mean absolute error was reduced to $1.4 \mathrm{~mm}$ (Fig. 4). The goal was to reduce this deviation to about $1 \mathrm{~mm}$. However, since this

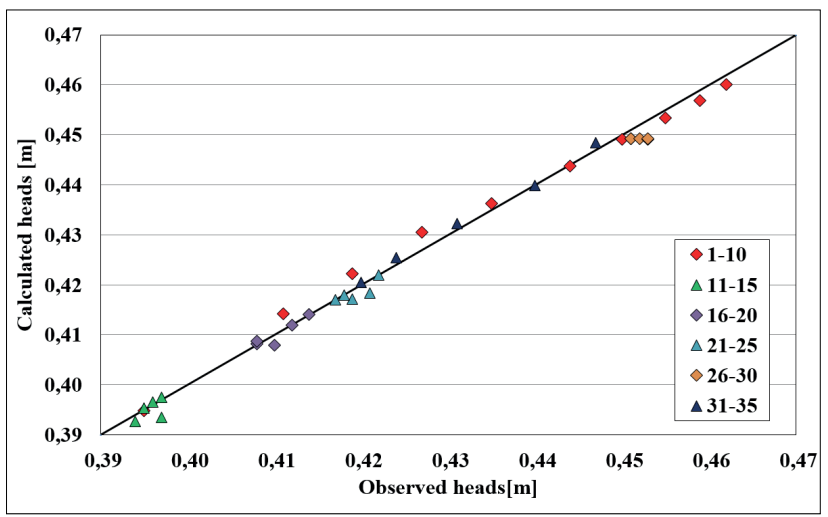

Fig. 4 Calibration results of the model variant FA 
would have been associated with the introduction of additional zones, the compromise was reached to accept the achieved accuracy of $1.4 \mathrm{~mm}$ and to waive further zones.

\section{Horizontally and vertically segmented model 10.1 Model variant $B$}

When calibrating the model variant $\mathrm{A}$, the main objective was to replicate the drawdown curve in the entire radial extent of the model more accurately. This was achieved at the expense of a detailed zoning of the model.

The physical model will also be used to study the cone of depression and to more accurately capture the drawdown curve in different soils. Based on this consideration, it was decided to create a model variant that only takes into account the 10 piezometers on the bottom plate, since in this case the aim was not to examine the immediate vicinity of the well. Consequently, in this case it is sufficient to use a numerical model with significantly fewer parameters.

Horizontally, only three zones were defined, passing through the ten model layers. The skin zone and zone I were preserved around the well. The third zone is the remaining model area (former zones II to V), which represents $99.8 \%$ of the model. Thus, a relatively simple and much more homogeneous model variant was defined as A. This model variant could be calibrated to the measured values of the piezometers 1 to 35 with an absolute average deviation of $2.8 \mathrm{~mm}$, to those of the piezometers 1 to 10 with a deviation of $1.4 \mathrm{~mm}$.

As described above, the simultaneous and very accurate calibration of both the well-near and well-remote piezometers is extremely difficult and almost impossible assuming homogeneous sand.

In this model variant, the calibration was focused on the piezometers 2 to 7 . In this model variant, a mean absolute error of $0.65 \mathrm{~mm}$ could be achieved with regard to these piezometers (Fig. 5).

\section{Distribution of the permeability coefficient in the FE model variants}

Table 2 summarizes the permeability coefficients of the individual zones in the model variants $\mathrm{A}$ and $\mathrm{B}$. In the variant $\mathrm{A}$, the model layers are denoted by L1 to L10.

Based on the values given in Tab. 2, the mean value weighted by the volumes of the zones can be calculated for the model variant $\mathrm{A}$ as $2.34 \times 10^{-4} \mathrm{~m} / \mathrm{s}$ and for the model variant $\mathrm{B}$ as $2.46 \times 10^{-4} \mathrm{~m} / \mathrm{s}$. Thus, considering the overall model, the deviation between the permeability coefficients of the two model variants is minimal despite the fact that

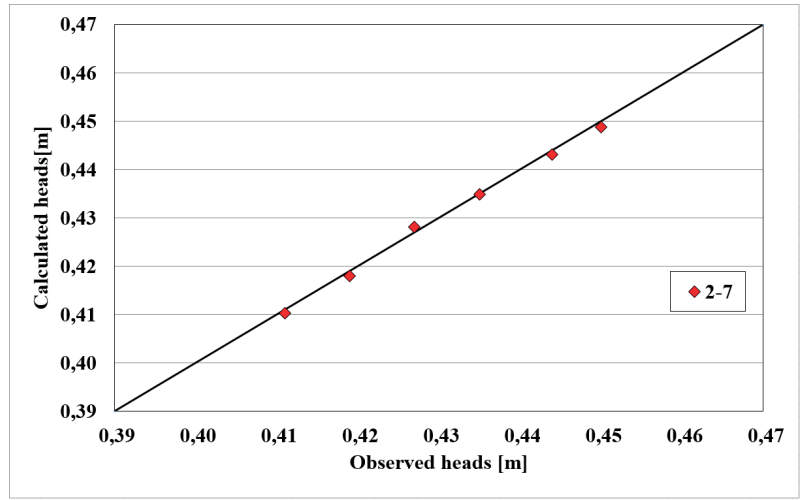

Fig. 5 Calibration results of the model variant B

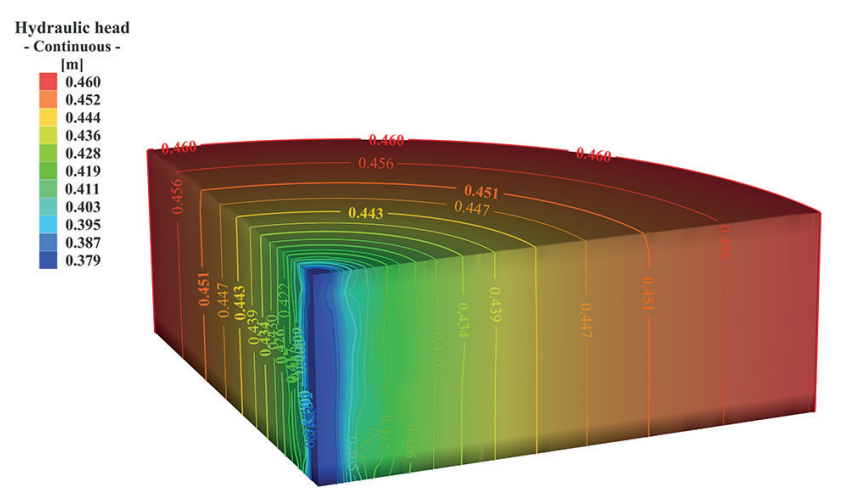

Fig. 6 Potential lines in the model variant A

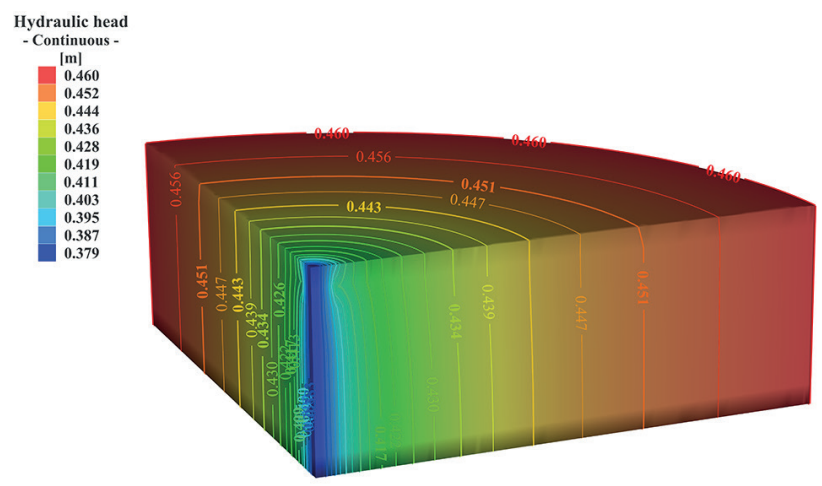

Fig. 7 Potential lines in the model variant B

the number of their parameters is substantially different. The model variant $A$ with a higher number of parameters more accurately reflected the water level as well as the local inhomogeneity occurring near the well due to the different permeabilities. This results in a very good agreement with the model variant $B$ for the permeability coefficient of the entire model space, which examines the process of the pumping test on a larger scale.

\section{Water levels in the calibrated model variants}

Fig. 6 shows the water levels and potential lines calculated in the model variant $\mathrm{A}$. The potential surfaces are cylindrically symmetric towards the model boundary, whereas in 
the direction of the well they are more distorted. The reason for this is that near the well, the zones of varying permeability become increasingly denser, as shown in Table 2 .

Fig. 7 shows the water levels and potential lines calculated in the model version B, whereby it is obvious that the potential lines in the entire model can be regarded as vertical, with the exception of the area close to the well. This is due to the fact that this model variant is much more homogeneous and a permeability change takes place only in the vicinity of the well.

\section{Validation of the model variants and limitations of the applicability of the physical model}

For both variant $\mathrm{A}$ and $\mathrm{B}$, data from a further 14 measurements were used to verify the accuracy of the calibration. Table 3 and 4 present the key figures of the validation for the model variants $\mathrm{A}$ and $\mathrm{B}$.

The correctness of the validation can be evaluated with three coefficients. Either the absolute mean deviation of the piezometers or the deviation between the flow rates or the ratio between the drawdown curve and the piezometer

Table 3 Results of the calibrated and validated model variant A

\begin{tabular}{|c|c|c|c|c|c|c|c|c|}
\hline \multirow{2}{*}{$\begin{array}{l}\text { Name of the } \\
\text { measurement }\end{array}$} & \multirow{2}{*}{$\begin{array}{l}\text { Drawdown } \\
{[\mathrm{mm}]}\end{array}$} & \multicolumn{2}{|c|}{ Boundary conditions [mm] } & \multirow{2}{*}{$\begin{array}{l}\text { Mean absolute } \\
\text { error from P2 to } \\
\text { P7 [mm] }\end{array}$} & \multirow{2}{*}{$\begin{array}{c}\text { Ratio piezometer } \\
\text { deviation and } \\
\text { drawdown [\%] }\end{array}$} & \multicolumn{2}{|c|}{ Discharge $[1 / \mathrm{min}]$} & \multirow{2}{*}{$\begin{array}{c}\text { Deviation of } \\
\text { the discharges } \\
{[\%]}\end{array}$} \\
\hline & & Pumping well & $\begin{array}{l}\text { Potential } \\
\text { boundary }\end{array}$ & & & Calculated & Measured & \\
\hline A & 81 & 379 & 460 & 1.4 & 1.7 & 0.21 & 0.21 & 0.0 \\
\hline $\mathrm{A}-\mathrm{V} 1$ & 181 & 299 & 480 & 3.8 & 2.1 & 0.42 & 0.40 & 5.2 \\
\hline $\mathrm{A}-\mathrm{V} 2$ & 162 & 318 & 480 & 3.2 & 2.0 & 0.39 & 0.37 & 4.1 \\
\hline $\mathrm{A}-\mathrm{V} 3$ & 142 & 338 & 480 & 2.9 & 2.0 & 0.35 & 0.34 & 1.9 \\
\hline A-V4 & 138 & 322 & 460 & 3.1 & 2.2 & 0.32 & 0.31 & 4.3 \\
\hline A-V5 & 124 & 356 & 480 & 2.9 & 2.4 & 0.31 & 0.30 & 3.3 \\
\hline A-V6 & 123 & 337 & 460 & 2.5 & 2.0 & 0.29 & 0.28 & 4.5 \\
\hline $\mathrm{A}-\mathrm{V} 7$ & 104 & 376 & 480 & 2.6 & 2.5 & 0.27 & 0.26 & 5.2 \\
\hline A-V8 & 79 & 381 & 460 & 1.8 & 2.3 & 0.20 & 0.20 & 2.6 \\
\hline A-V9 & 67 & 413 & 480 & 3.0 & 4.4 & 0.19 & 0.19 & 1.2 \\
\hline A-V10 & 59 & 401 & 460 & 2.4 & 4.0 & 0.16 & 0.15 & 4.6 \\
\hline A-V11 & 46 & 434 & 480 & 3.3 & 7.1 & 0.13 & 0.15 & 12.4 \\
\hline $\mathrm{A}-\mathrm{V} 12$ & 38 & 422 & 460 & 2.7 & 7.2 & 0.11 & 0.12 & 13.0 \\
\hline A-V13 & 25 & 455 & 480 & 2.8 & 11.3 & 0.07 & 0.10 & 28.3 \\
\hline $\mathrm{A}-\mathrm{V} 14$ & 14 & 446 & 460 & 2.4 & 17.0 & 0.04 & 0.07 & 58.8 \\
\hline
\end{tabular}

Table 4 Results of the calibrated and validated model variant B

\begin{tabular}{|c|c|c|c|c|c|c|c|c|}
\hline \multirow{2}{*}{$\begin{array}{l}\text { Name of the } \\
\text { measurement }\end{array}$} & \multirow{2}{*}{$\begin{array}{c}\text { Drawdown } \\
{[\mathrm{mm}]}\end{array}$} & \multicolumn{2}{|c|}{ Boundary conditions [mm] } & \multirow{2}{*}{$\begin{array}{l}\text { Mean absolute } \\
\text { error from P2 to } \\
\text { P7 [mm] }\end{array}$} & \multirow{2}{*}{$\begin{array}{c}\text { Ratio piezometer } \\
\text { deviation and } \\
\text { drawdown [\%] }\end{array}$} & \multicolumn{2}{|c|}{ Discharge $[1 / \mathrm{min}]$} & \multirow{2}{*}{$\begin{array}{c}\text { Deviation of } \\
\text { the discharges } \\
{[\%]}\end{array}$} \\
\hline & & Pumping well & $\begin{array}{l}\text { Potential } \\
\text { boundary }\end{array}$ & & & Calculated & Measured & \\
\hline B & 81 & 379 & 460 & 0.6 & 0.8 & 0.21 & 0.21 & 0.0 \\
\hline B-V1 & 181 & 299 & 480 & 2.1 & 1.1 & 0.43 & 0.40 & 6.4 \\
\hline B-V2 & 162 & 318 & 480 & 2.0 & 1.2 & 0.39 & 0.37 & 5.6 \\
\hline $\mathrm{B}-\mathrm{V} 3$ & 142 & 338 & 480 & 2.3 & 1.6 & 0.35 & 0.34 & 3.3 \\
\hline B-V4 & 138 & 322 & 460 & 1.4 & 1.0 & 0.33 & 0.31 & 5.1 \\
\hline B-V5 & 124 & 356 & 480 & 2.3 & 1.8 & 0.31 & 0.30 & 4.4 \\
\hline B-V6 & 123 & 337 & 460 & 1.5 & 1.3 & 0.30 & 0.28 & 5.6 \\
\hline B-V7 & 104 & 376 & 480 & 1.9 & 1.8 & 0.27 & 0.26 & 5.1 \\
\hline B-V8 & 79 & 381 & 460 & 1.2 & 1.5 & 0.20 & 0.20 & 2.8 \\
\hline B-V9 & 67 & 413 & 480 & 2.1 & 3.2 & 0.18 & 0.19 & 2.6 \\
\hline B-V10 & 59 & 401 & 460 & 1.1 & 1.9 & 0.15 & 0.15 & 2.0 \\
\hline B-V11 & 46 & 434 & 480 & 1.5 & 3.3 & 0.13 & 0.15 & 18.4 \\
\hline B-V12 & 38 & 422 & 460 & 1.4 & 3.8 & 0.10 & 0.12 & 19.0 \\
\hline B-V13 & 25 & 455 & 480 & 1.5 & 5.8 & 0.07 & 0.10 & 35.0 \\
\hline B-V14 & 14 & 446 & 460 & 1.2 & 8.3 & 0.04 & 0.07 & 70.1 \\
\hline
\end{tabular}


error can be considered. This is necessary to determine in which interval the physical model provides reliable results in terms of the drawdown. If the maximum deviations of the piezometers are compared, the model variant A with $3.8 \mathrm{~mm}$ (Table 3) has a larger maximum than the model variant B with $2.3 \mathrm{~mm}$ (Table 4).

Furthermore, the lower ratio results in at the same piezometer deviations and at the greater drawdown. The deviation between the flow rates measured in the physical model and those calculated in the numerical model is an important indication in the evaluation of the model results. Looking closely at these coefficients, it is possible to determine in which ranges the physical model provides reliable results.

After evaluating the data given in Tab. 3, two categories can be defined depending on how large the ratio between the piezometer deviation and the drawdown and that between the flow rates. Both categories have been assigned to a drawdown range, in which the findings made here are valid. These categories are "unusable" and "reliable".

If the rounded values of the two coefficients in both model variants exceed $5 \%$, the results supplied by the physical model are considered unusable in this drawdown range. This criterion applies to the model variant $\mathrm{A}$ for the measurements A-V11, A-V12, A-V13 and A-V14 (Table 3 ) as well as the model variant $B$ for the measurements B-V11, B-V12, B-V13 and B-V14 (Table 4).

Consequently, if the drawdown remains below $50 \mathrm{~mm}$, the results are unacceptable. The larger deviations may result from the laboratory measurements being subject to a small but constant error, which appears more pronounced for smaller drawdown and flow rates. It is also conceivable that the conditions that provide the similarity of the investigated process are violated by the scale effect at such small potential differences.

Based on the other data in Tables 3 and 4, it can be seen that the physical model provides acceptable results in the drawdown interval between 50 and $181 \mathrm{~mm}$.

\section{Conversion of the model tests results to real sizes}

As explained above, the well diameter in the original model had to be increased from $10 \mathrm{~mm}$ to $17 \mathrm{~mm}$. As a result, the model scale changed from $\lambda=16$ to $\lambda=9.4$.

By applying the length scale $\lambda=9.4$, the drawdown and flow rate quantities obtained in the physical model can be converted into real variables occurring in nature (prototype). Table 5 summarizes the quantities obtained in the physical and numerical model and the converted ones. The calculation of the real variables such as drawdown and flow rate took place according to the model laws (Tab. 1).

The drawdown measured in the physical model is to be multiplied by $\lambda$, the flow rate measured by $\lambda^{2}$ to obtain the field quantities [16]. Furthermore, it should be noted that the physical model has the shape of a quarter-cylinder, therefore, the flow rates measured in the model have to be multiplied by 4 [18].

If the mean absolute error of the piezometers in the numerical model is multiplied by $\lambda$, the accuracy of the drawdown in the field scale is calculated. The accuracy of the flow rates was calculated so that the flow rates determined for the real quantities were multiplied by the errors obtained in the numerical model variants. The error of water levels and flow rates can have a positive or negative sign, thus the physical model under- or overestimates the exact values with the values given in Table 4. Neither the accuracy of the flow rates nor those of the water levels is dependent on the drawdown. So when the drawdown increases, it does not necessarily follow that the error of the flow rate or the water levels also increases.

As noted in Table 3 and 4, the model does not provide reliable results when the drawdown is below $50 \mathrm{~mm}$ in the physical model. If this value is multiplied by $\lambda=9.4$, the result is $470 \mathrm{~mm}$. In practice, it can be stated that the model can be used for drawdown over approx. $0.5 \mathrm{~m}$.

The water levels calculated in model A are less accurate than those of model B. During calibration, however, the difference between the mean absolute errors was twice as large because of the different number of piezometers. Since the model A describes in more detail and with greater accuracy the water level and the distribution of the permeability coefficients, the flow rates obtained with it can be considered more accurate.

The flow rate values converted into real sizes shown in Table 5 were also checked analytically. Using the measurements of the observation wells, the flow rates were calculated according to the Dupuit method. For permeability coefficient, the value calibrated in model B was assumed to be $2.46 \times 10^{-4} \mathrm{~m} / \mathrm{s}$, because this model can be regarded as homogeneous. The results are summarized in Table 6. The flow rate values of the physical model, which are based on a quarter-cylinder, were converted to the total cylinder with the multiplier 4 in order to be able to compare them with the values calculated on the basis of the Dupuit formula. Subsequently, both the analytically calculated values $\left(Q_{a}\right)$ and the values measured in the physical model $\left(\mathrm{Q}_{\mathrm{m}}\right)$ were multiplied by the conversion factor $\lambda^{2}$. 
Table 5 Conversion of the model tests results into real field quantities

\begin{tabular}{|c|c|c|c|c|c|c|c|c|c|c|c|c|}
\hline \multirow{3}{*}{ 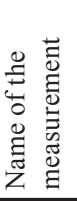 } & \multicolumn{2}{|c|}{$\begin{array}{c}\text { Numerically calculated } \\
\text { values }\end{array}$} & \multicolumn{4}{|c|}{ Values converted to real field quantities } & \multirow{3}{*}{$\begin{array}{l}\text { Drawdown } \\
{[\mathrm{mm}]}\end{array}$} & \multicolumn{5}{|c|}{ Values measured in the physical model } \\
\hline & \multirow{2}{*}{$\begin{array}{l}\text { Drawdown } \\
{[\mathrm{mm}]}\end{array}$} & \multirow{2}{*}{$\begin{array}{l}\text { Discharge } \\
{[1 / \mathrm{min}]}\end{array}$} & \multicolumn{2}{|c|}{$\begin{array}{c}\text { Deviation } \\
\text { piezometer }[\mathrm{mm}]\end{array}$} & \multicolumn{2}{|c|}{$\begin{array}{c}\text { Deviation } \\
\text { discharge }[\%]\end{array}$} & & \multirow{2}{*}{$\begin{array}{l}\text { Discharge } \\
{[1 / \mathrm{min}]}\end{array}$} & \multicolumn{2}{|c|}{$\begin{array}{l}\text { Accuracy water } \\
\text { level }[\mathrm{mm}]\end{array}$} & \multicolumn{2}{|c|}{$\begin{array}{c}\text { Accuracy } \\
\text { discharge }[1 / \mathrm{min}]\end{array}$} \\
\hline & & & A & $\mathrm{B}$ & A & B & & & A & $\mathrm{B}$ & A & $\mathrm{B}$ \\
\hline A & 81 & 0.21 & 1.4 & - & 0.0 & - & 762 & 74 & 13.3 & - & 0 & - \\
\hline B & 81 & 0.21 & - & 0.6 & - & 0.0 & 762 & 74 & - & 6.1 & - & 0 \\
\hline V1 & 181 & 0.40 & 3.8 & 2.1 & 5.2 & 6.4 & 1703 & 142 & 36.0 & 19.3 & 7.4 & 9.1 \\
\hline V2 & 162 & 0.37 & 3.2 & 2.0 & 4.1 & 5.6 & 1524 & 131 & 30.0 & 18.6 & 5.4 & 7.3 \\
\hline V3 & 142 & 0.34 & 2.9 & 2.3 & 1.9 & 3.3 & 1336 & 120 & 27.3 & 21.4 & 2.3 & 4.0 \\
\hline V4 & 138 & 0.31 & 3.1 & 1.4 & 4.3 & 5.1 & 1299 & 110 & 28.9 & 13.3 & 4.7 & 5.6 \\
\hline V5 & 124 & 0.30 & 2.9 & 2.3 & 3.3 & 4.4 & 1167 & 106 & 27.6 & 21.3 & 3.5 & 4.7 \\
\hline V6 & 123 & 0.28 & 2.5 & 1.5 & 4.5 & 5.6 & 1157 & 99 & 23.2 & 14.6 & 4.5 & 5.6 \\
\hline V7 & 104 & 0.26 & 2.6 & 1.9 & 5.2 & 5.1 & 979 & 92 & 24.2 & 17.8 & 4.8 & 4.7 \\
\hline V8 & 79 & 0.20 & 1.8 & 1.2 & 2.6 & 2.8 & 743 & 71 & 17.4 & 11.0 & 1.8 & 2.0 \\
\hline V9 & 67 & 0.19 & 3.0 & 2.1 & 1.2 & 2.6 & 630 & 67 & 27.8 & 20.1 & 0.8 & 1.7 \\
\hline V10 & 59 & 0.15 & 2.4 & 1.1 & 4.6 & 2.0 & 555 & 53 & 22.4 & 10.4 & 2.4 & 1.1 \\
\hline
\end{tabular}

From these flow rates, the ratio $Q_{a} / Q_{m}$ was determined, the average value was 0.90 . This goodness of agreement between the two methods is found to be acceptable. However, e.g. in the case of dewatering of foundation pits, where the most accurate estimation of the discharge quantity is the primary task for the most economical design, a deviation of $10 \%$ represents a significant additional cost. With the calibrated and validated numerical models of different numbers of parameters (A and B), the measurements in the physical model were verified and almost identical permeability coefficients were obtained.

\section{Results and conclusions}

Our physical model was validated by two model variants implemented in the FE software Feflow. In the model variant $\mathrm{A}$, the water level and the flow conditions in the entire model domain, but in the immediate vicinity of the well were described with the greatest accuracy. In the model variant $B$ the spreading of the drawdown in the well was examined. In that case, the immediate environment of the well should not be described, so the number of parameters to be calibrated has been reduced.

For both model variants, the total permeability coefficient of the soil was calculated based on the total model volume, weighted with the volumes of the individual zones. Between the permeability coefficients of the two model variants, a minimal deviation was obtained. Therefore, it can be stated that the model with a lower number of parameters is sufficient for determining the permeability coefficient of the entire model.
The potential lines of the model variants point towards the well an increasing deviation. The reason for this is the difference in the distribution of the permeability coefficient. So the difference in the flow pattern becomes apparent when the permeability in the well-near area is defined more precisely. Thus, to describe the immediate environment of the well, the higher number of parameters and a complex model is required.

Both model variants were validated with further independent measurements and then the results were converted into field quantities according to the model laws. From the results, it can be seen that the experiments performed in the physical model provide the well discharge in the medium sand with acceptable accuracy when the real drawdown varies between 0.5 and 1.7 meters.

The discharges converted to real quantities were also analytically tested on the basis of the model B. The mean of the deviations between the numerical and analytical methods is $10 \%$. Accordance at this level is considered acceptable. Since the solution to a pit drainage problem is the more economical the more accurately the discharge can be estimated. In this case, a deviation of $10 \%$ of the discharges can also represent significant additional costs. With the models of different parameter numbers A and B, the measurements of the physical model were validated and almost identical permeability coefficients obtained.

Based on the investigations, it can be seen that the distorted physical model is suitable for studying the pumping process in a particular soil, whereby the lowered water level, the distribution of the permeability coefficient and 
the cone of depression can be analysed at different drawdown and flow rates. The measurement results of the physical model can be converted to real quantities, which can provide an alternative way of approaching the field studies.

Measurements in the physical model can help in the planning phase with excavation pit dewatering and the utilisation of groundwater bodies. Recommendations may be given based on the estimate of the cone of depression for the optimal number and location of the observation wells. A good approximation for the pretest can be obtained to determine the maximum flow of the pumping test. Due to the cone of depression in the physical model can be closed to the stationary water level of the well in the field, so the time required for the pumping test can also be estimated.

The investigations in the physical model should be extended with other types of soil. It is intended to investigate not only granular but also cohesive as well as layered soils. Of particular interest are the lowering conditions around partially penetrated wells. Experience has shown that testing the measurement results in a numerical model is indispensable. Therefore, with whatever configuration the experiments are continued, the numerical simulation of the model tests is made at the same time.

\section{Acknowledgement}

The authors would like to thank DHI-WASY for providing a single-seat license of FEFLOW 7.0 to prepare the dissertation of Mr. Dávid Farkas.

Support of grant BME FIKP-VÍZ by EMMI is kindly acknowledged.

\section{References}

[1] Committee for Standardization "ISO 22282:2012 Geotechnical investigation and testing - Geohydraulic testing", Part 1 to Part 6, CEN, Brussels, Belgium, 2012.

[2] Busch, K.-F., Luckner, L., Tiemer, K. "Geohydraulik, Lehrbuch der Hydrologie" (Geohydraulics, Textbook of Hydrology), 3rd ed., Gebrüder Bornträger, Berlin, Stuttgart, 1993. (in German)

[3] Blau, R. V., Trüeb, E., Fisch, W., Hufschmid, P. "Grundlagen für Schutz und Bewirtschaftung der Grundwasser des Kantons Bern" (Basics for protection and management of the groundwater of the canton of Berne), Wasser-u. Energiewirtschaftsamt des Kantons Bern, Bern, Switzerland, 1983. (in German) [online] Available at: https://www. bve.be.ch/bve/de/index/umwelt/umwelt/geologie/Geologische Dokumentationsstelle/Grundlagenberichte.assetref/dam/documents/ BVE/AWA/de/UMWELT/Geologie/Grundlagenberichte/Hydrogeo logie_Emmental_Teil_IV_Modellstudie.pdf [Accessed: 20.02.2019]

[4] Korom, S. F., Bekker, K. F., Helweg O. J. "Influence of Pump Intake Loacation of Well Efficiency", Journal of Hydrologic Engineering, 8(4), pp. 197-203, 2003.
https://doi.org/10.1061/(ASCE)1084-0699(2003)8:4(197)

[5] Simpson, M. J., Clement, T. P., Gallop, T. A. "Laboratory and numerical investigation of flow and transport near a seepage-face boundary", Ground Water, 41(5), pp. 690-700, 2003. https://doi.org/10.1111/j.1745-6584.2003.tb02407.x

[6] Klauder, W. S. "Experimentelle Untersuchung der Anströmung von Vertikalfilterbrunnen", Doctoral dissertation, RheinischWestfälischen Technischen Hochschule, Aachen, 2010. (in German) [online] Available at: http://publications.rwth-aachen.de/ record/63946/files/3570.pdf [Accessed: 20.02.2019]

[7] Davidenkoff, R., Franke, L. "Räumliche Sickerströmung in eine umspundete Baugrube im Grundwasser" (Spatial infiltration into a submerged excavation in groundwater), Die Bautechnik, 12, pp. 108-116, 1966. (in German) [online] Available at: https://izw.baw. de/publikationen/mitteilungsblaetter/0/mb24_Davidenkoff_Franke. pdf [Accessed: 20.02.2019]

[8] Schnitter, G., Zeller, J. "Sickerströmung als Folge von Stauspiegelschwankungen in Erddämmen" (Leakage flow as a result of congestion level fluctuations in earth dams), Schweizerische Bauzeitung, 75,(52) pp. 808-814, 1957. (in German)

[9] Schincariol, R. A., Schwartz, F. W. "An experimental investigation of variable density flow and mixing in homogeneous and heterogeneous media", Water Resources Research, 26(10), pp. 2317-2329, 1990 .

https://doi.org/10.1029/WR026i010p02317

[10] Stoeckl, L., Walther, M., Graf, T. "A new numerical benchmarks of a freshwater lens", Water Resources Research, 52(4), pp. 2474-2489, 2016. https://doi.org/10.1002/2015WR017989

[11] Karay, Gy., Hajnal, G. "Modelling Methods Attached to the Research of Groundwater Flow in Fractured Rocks - Theories, Laboratory and Numerical Modelling", Periodica Polytechnica Civil Engineering, 60(3), pp. 437-448, 2016.

https://doi.org/10.3311/PPci.8661

[12] Faulkner, J., Hu, B. X., Kish, S., Hua, F. "Laboratory analog and numerical study of groundwater flow and solute transport in a karst aquifer with conduit and matrix domains", Journal of Contaminant Hydrology, 110(1-2), pp. 34-44, 2009. https://doi.org/10.1016/j.jconhyd.2009.08.004

[13] Wu, Y., Hunkeler, D. "Hyporheic exchange in a karst conduit and sediment system - A laboratory analog study", Journal of Hydrology, 501, pp. 125-132, 2013. https://doi.org/10.1016/j.jhydrol.2013.07.040

[14] Ivicsics, L. "Hydraulic models", 1st ed., Water Resources Publications, Fort Collins, Colorado, United States, 1980.

[15] Kovács, Gy. "Seepage hydraulics", 1st ed., Development in water science 10, Elsevier Science, Amsterdam, Netherlands, 1981.

[16] Mosonyi, E., Kovács, Gy. "Loi de modèles réduits de filtration" (Act of reduced models of filtration), Symposia Darcy, IAHS, Dijon, France, pp. 111-115, 1956. (in French)

[17] Barta, E., Hajnal, G., Veczán, É., Vasvári, V. "Bestimmung des Durchlässigkeitsbeiwertes mithilfe von Modellversuchen" (Determination of the permeability coefficient using model tests), In: Zenz, G. (ed.): Wasserbausymposium 2012, Graz, Austria, 2012, pp. 307-314. (in German) 
[18] Szabó, G., Hajnal, G., Vasvári, V. "Inverse numerische Modellierung zur Bestimmung des Durchlässigkeitsbeiwertes" (Inverse numerical modeling to determine the permeability coefficient), In: Zenz, G. (ed.): Wasserbausymposium 2012, Graz, Austria, 2012, pp. 233240, (in German)

[19] Barta, E., Hajnal, G., Karay, Gy., Vasvári, V. "Determination of the Coefficient of Permeability by Physical Model test and Numerical Modelling", In: 35th IAHR Congress 2013, Beijing, China, 2013, pp. 3236-3246.

[20] Klotz, D. "Untersuchung von Grundwasser-strömung durch Modellversuche im Maßstab 1:1" (Investigation of groundwater flow through model experiments on a scale of 1:1), Geologica Bavarica, 64, pp. 75-119. 1971. (in German)
[21] Diersch, H-J. G. "FEFLOW - Finite Element Modeling of Flow, Mass and Heat Transport in Porous and Fractured Media", Springer, Berlin, Heidelberg, Germany, 2014. https://doi.org/10.1007/978-3-642-38739-5

[22] DHI, MIKE, FEFLOW 7.0, User Guide, DHI-WASY, Berlin, Germany, 2015. [online] Available at: http://www.softfactory. kr/download/feflow/FEFLOW_70_user_manual.pdf [Accessed: 20.02.2019] 